\title{
Traditional Chinese medicine herbs are they safe for psoriatic patients?
}

\section{Joanna Patrycja Bartosińska ${ }^{1}$, Aldona Pietrzak ${ }^{1}$, Jacek Szepietowski ${ }^{2,3}$, Jacob Dreiher ${ }^{4}$, Ryszard Maciejewski ${ }^{5}$, Grażyna Chodorowska ${ }^{1}$}

\author{
${ }^{1}$ Department of Dermatology, Venereology and Pediatric Dermatology, Medical University \\ of Lublin, Poland \\ ${ }^{2}$ Department of Dermatology, Venereology and Allergology, Medical University, Wroclaw, Poland \\ ${ }^{3}$ Ludwik Hirszfeld Institute of Immunology and Experimental Therapy, Polish Academy of Sciences, \\ Wroclaw, Poland \\ ${ }^{4}$ Division of Health in the Community, Ben Gurion University of the Negev, Beer Sheva, Israel \\ ${ }^{5}$ Department of Human Anatomy, Medical University of Lublin, Poland
}

\begin{abstract}
Although traditional Chinese medicine (TCM) relies on remedies of natural origin, its use is not always safe as it can have not only beneficial but also deleterious effects. Psoriatic patients, disappointed by conventional treatment and unaware of the potential side effects of TCM preparations, are increasingly reaching for non-traditional therapeutic methods. This review presents brief characteristics of selected Chinese herbs self-prescribed by psoriatic patients. It is important that dermatologists should be able to recognize any potential hazards connected with current or previous taking of these herbs by their patients. (Folia Histochemica et Cytobiologica 2011, Vol. 49, No. 2, 201-205)
\end{abstract}

Key words: traditional Chinese medicine, Chinese herbs, herbal medicine, psoriasis, adverse reaction

\section{Introduction}

As a chronic and incurable disease, psoriasis is characterized by periods of remission and exacerbation resulting from endogenous or exogenous factors. This debilitating condition negatively affects the lives of patients and is the main cause of any psychological and psychosocial problems that they develop. Currently applied methods of treatment are still unsatisfactory, and being burdensome or unpleasant, they often do not meet the patients' expectations. Even the presently recommended biological treatment appears to be limited to treating more severe psoriatic cases and, because of its very high cost, cannot be routinely used in many countries.

Correspondence address: J.P. Bartosińska, Department of Dermatology, Venereology and Pediatric Dermatology,

Medical University of Lublin, Poland;

Radziwillowska Str. 13; 20-080 Lublin;

e-mail: jbartosinski@gmail.com
Therefore, observing no immediate results, many patients become discouraged or frustrated and start looking for alternative means of treatment. Traditional Chinese medicine (TCM) is one option. However, the assumption that herbal preparations used in TCM, known as Chinese materia medica (CMM), are safe for human use (because of their natural origin), is a common misconception [1-3]. According to TCM proponents, this kind of treatment typically involves the application of herbs and vitamins as well as the use of a wide range of therapeutic methods, such as massages, acupuncture, diet and lifestyle modification [3].

This paper attempts a short study of the characteristics of Chinese herbs available on the market and frequently found in herbal therapeutic mixtures.

\section{Pros and cons of TCM}

CMMs are believed to have a positive influence on various pathogenic mechanisms observed in pso- 
riasis because of their anti-inflammatory and antiangiogenic effects, as well as their potential to change the cytokine balance. Thus, the use of TCM herbal preparations containing e.g. Indigo naturalis, Rhizoma smilcis glabre, Radix angelicae dahuricae, Radix salviae milthiorrhiza, Radix rehmanniae glutinosae, Radix angelicae sinensis, Dictamnus dasycarpus, Lithospermum erythrorhizon, Radix paeoniae lactiflorae, Radix rubiae, Flos carthami tinctorii and Radix glycyrrhizae uralensis, seems to be justified in the treatment of psoriatic skin changes [1, 4]. According to the traditional Chinese medicine practitioners, these herbs relieve the symptoms typical of psoriasis and restore the internal bodily balance [1]. But, despite their natural origin, CMMs do not appear to be entirely safe, as they have been reported to bring about adverse reactions including hypersensitivity, hepatotoxicity, renal failure, adult respiratory distress syndrome, cardiomyopathy and various skin problems, such as allergic contact dermatitis $[3,5,6]$.

Easy access to TCM preparations (available as OTCs) makes them popular among patients. One study, for instance, revealed that $51 \%$ of psoriatic patients opted to use alternative therapies [7].

Although the demand for TCM preparations seems to be great and ever growing, scientific evidence confirming their efficacy is scarce in European medical journals. In fact, almost all reports on the TCM preparations' modes of action are found in Chinese medical literature. To date there have been no randomized, placebo-controlled clinical trials, which could confirm their safety and efficacy $[8,9]$.

TMMs are predominantly intended for external use, although some are oral or parenteral preparations [8]. But, even though they are usually a mixture of many ingredients, detailed information concerning their composition and dosage is usually hard to find, because TCM herbal preparations are often sold in unlabeled containers and packages [10]. More often than not, the composition of each medication is decided by Chinese medicine practitioners who take into consideration the type of ailment and specific symptoms presented by individual patients. For example, according to TCM practitioners, psoriasis is divided into three main categories: a 'blood-heat' type, 'blood-deficiency-dryness' type and 'blood stasis' type. Hence, the composition of medications meant for psoriatic patients is decided individually on the basis of the suspected type of psoriasis [3].

Having observed some improvement, patients tend to notice only the beneficial effects of the applied medications, and not to realize the side effects the medications may cause. Therefore, clinicians should be on the lookout for any adverse reactions in their patients [8].

\section{Characteristics of selected TCM herbal preparations used by psoriatic patients}

One of the best studied herbs in the treatment of psoriatic patients, Indigo naturalis, is a dark-blue powder prepared from the leaves of plants such as Baphicacavthus cusia, Polygonum tinctorium, Isatis indigotica and Indigofera tinctoria. Its major active components are indirubin, indigo, and tryptanthrin. According to the literature reports, Indigo naturalis exhibits antiviral, antibacterial and anti-tumor properties $[9,11]$. TCM uses this herb in the treatment of various inflammatory and infectious diseases e.g. eczema, aphthae, skin eruptions, mumps, erysipelas, herpes zoster, carbuncles and furuncles. Yet, because of its poor solubility in water and poor absorption, its use is limited [9, 11, 12]. Indigo naturalis' ability to modify the proliferation and differentiation of keratinocytes explains its application in the treatment of psoriatic patients. Moreover, it is thought to exert an anti-inflammatory effect and induce apoptosis $[9,12]$. Immunohistochemical studies conducted by Lin et al. [9], who investigated the effect of Indigo naturalis on human psoriatic skin lesions, suggest that this herb effectively decreases the level of proliferating cell nuclear antigen, and increases the level of involucrin, a known differentiation marker. Thus, this sort of treatment may restore the physiological balance in the epidermis. Moreover, indirubin has been shown to inhibit the production of interferon- $\gamma$, interleukin-6 and RANTES chemokine, also involved in the pathogenesis of psoriasis [12].

Indigo naturalis may be administered both topically and by mouth. However, the long-term systemic application of this herb has been proven to cause gastrointestinal problems and liver damage. Adverse gastrointestinal reactions following Indigo naturalis administration have been reported in the literature and their incidence may range from $26 \%$ to even $96 \%$. Some of them have even been reported as 'severe' $[3,8]$.

Temporary reductions in peripheral white blood cell (WBC) counts following Indigo compounds' application have been previously reported [3].

According to practitioners of Chinese medicine, topical application of Indigo naturalis-containing preparations appears to be safer since it prevents potential systemic side effects. This opinion is supported by the study of Lin et al. [13], in which approximately $74 \%$ of the patients treated with an indigo naturalis-containing ointment experienced clearance or near-clearance of psoriatic lesions. In none of these patients were serious adverse events encountered. However, four patients reported transient itching after application of the ointment. 
In another study by Lin et al. [14] about $95 \%$ reduction in the PASI score (Psoriasis Area Severity Index) and TBSA (total body surface area) after application of Indigo naturalis ointment were noted. They reported no allergic reactions or dermatitis in the studied psoriatic patients. The patients' blood parameters as well as liver and renal function tests were within the normal range. The same authors' molecular study revealed decreased expressions of $\mathrm{Ki}-67$ proliferating marker as well as CD3 inflammatory marker, whereas filaggrin, the differentiation marker, was increased in the epidermis [11].

According to TCM, Rhizoma smilcis glabre prevents leptospirosis and is supposed to be helpful in the treatment of syphilis, brucellosis, acute bacterial dysentery, acute and chronic nephritis and even AIDS $[15,16]$. The herb has an anti-tumor, anti-inflammatory, immunomodulatory and anti-atherosclerotic effect $[15,17]$. The study by Zhang et al. [17] demonstrated its antioxidant properties which result from the activity of astilbin, a flavonoid contained in Rhizoma smilcis glabre. Thus, Rhizoma smilcis glabre is claimed to relieve various skin disorders, including psoriasis [16, 17]. On an animal model, Rhizoma smilcis glabre, as well as Dictamnus dasycarpus (another herb with anti-psoriatic potency) selectively inhibit delayed type hypersensitivity response with no influence on humoral immune reactions. This mechanism involves selective stimulation of apoptosis of activated $\mathrm{T}$ cells and has no impact on naive $\mathrm{T}$ cells and other tissue cells $[1,18]$. Jiang et al. [15], in their study conducted on rats, pointed out that Rhizoma smilcis glabre may also have a direct anti-inflammatory effect accomplished by inhibiting the synthesis or release of prostaglandin E2. It is thought that both herbs have a hepatoprotective effect and are even capable of reversing some liver abnormalities and may reduce potentially toxic effects of other herbs $[1,18]$. Moreover, Rhizoma smilcis glabre, by a selective suppression on lymphocyte function via reducing matrix metalloproteases and nitric oxide production, may be effective in relieving stiff joints [19].

Radix Angelicae dahuricae is regarded as an effective agent in the treatment of acne, erythema, headache, toothache, sinusitis, colds and flu [20]. It contains some furocumarins, including imperatorin, isoimpertorin and alloimperatorin, actively involved in the inhibition of both cyclooxygenase and lipoxygenase pathways of arachidonate metabolism [8, 21]. Since Angelica species are a source of psoralens, they are applied both systemically and topically, being also a substitute for the PUVA-therapy. In the study by Chen et al. [5] some patients treated with the herb presented allergic photosensitivity reactions.
Radix salviae milthiorrhiza contains a variety of diterpenoids, phenolics, flavonoids, triterpenoids and sterols. Its active components have anti-ischamic, antioxidant and anti-tumor activity [22]. Salvia miltiorrhiza is known to have a significant inhibitory effect on the production of IFN- $\gamma$ from lymphocytes and Il-12 from macrophages as well as suppression of mast cell degranulation [1]. This herb is thought to exhibit some hepatoprotective effect which is in part due to its ability to improve blood circulation and promote regeneration of the liver cells [23].

Glycyrrhiza uralensis, another Chinese herb which may have a protective effect on hepatocytes and gastrointestinal tract, is commonly used as a component of various anti-psoriatic herbal preparations. Therefore, it is believed to prevent side effects commonly observed in the process of herbal therapy application. Moreover, Glycyrrhiza uralensis exerts both immunosuppressive and immuno-enhancing activities, which may modulate abnormal immunological processes in psoriasis [1].

Rehmannia glutinosa, a frequent herbal mixtures' ingredient, is a Chinese herb used in the treatment of psoriasis as well as various skin rashes [1]. On their animal model, Kim et al. [24] have found that the root of Rehmannia glutinosa is capable of inhibiting the release of histamine and production of TNF- $\alpha$ and Il-1, which may have a positive effect on the treatment of psoriatic patients.

Radix rubiae (Rubia cordifolia L.) is commonly prescribed in Chinese herbal medicine, especially in febrile and bleeding conditions [25]. Because of its anti-proliferative activity, it is also used as an ingredient of various anti-psoriatic Chinese herb mixtures. Tse et al. [26], in their study on cultured $\mathrm{HaCaT}$ cells, found that some herbs, including the root of Rubia cordifolia, have a significant anti-proliferative effect due to inducing cell cycle arrest at the G1 phase. The herb is capable of causing apoptosis of the HaCaT keratinocytes in a time- and concentration-dependent manner. The authors have not observed cytotoxicity to the human fibroblast cell line. Moreover, it has been revealed that Radix rubiae has many other biological properties, including anti-oxidant, immunosuppressive, hemostatic, white blood cell count-boosting and anti-cancer activities [25].

Another Chinese herb, Flos carthami tinctorii, can inhibit the production of TNF- $\alpha$, Il- $1 \alpha$, Il- $1 \beta$ and Il-6 from stimulated human monocytes. Similarly, Radix lithospermi erythrorhizoni, due to its inhibitory effect on TNF- $\alpha$-induced angiogenesis and suppression of Il- $1 \alpha$ production, is thought to have a positive influence on the course of psoriasis, although scientific evidence concerning the safe use of the latter plant is still ambiguous [1]. 
Finally, according to TCM practitioners, Radix paeoniae lactiflorae may be beneficial in psoriatic arthritis [1].

\section{Potential side effects of TCM preparations}

Even though TCM proponents continue to come up with new evidence supporting the efficacy of TCM medications, their side effects are seldom brought to light. This may result in patients' unawareness of dire consequences of taking them without consulting their doctor. Patients are sometimes unable to see or understand the link between taking TCM medicines and their deteriorating condition. It is obvious that at least some patients who take TCM medications of their own accord will seek a doctor's assistance at some point of their 'self-prescribed treatment'.

Adverse reactions to Chinese medicines can develop due to response to natural compounds, natural toxins or to contaminants (pesticides, lead, mercury, arsenic, diazepam, thiazide diuretics, ephedrine and caffeine) $[2,10,27]$. Thus, side effects have been divided into five types: unpredictable adverse effects (AEs), AEs arising from improper use, AEs arising from contamination, AEs arising from misidentification of CMMs, and AEs arising from drug/herb interaction [2].

Adverse reactions during TCM treatment range from mild (transient hepatitis, dermatitis) to severe fatal illnesses (anaphylactic shock, liver failure) [10]. Liver complications, including elevated liver enzymes, are one of the best documented adverse effects of TCM preparations [3], whereas allergic contact dermatitis is a commonly observed skin side effect to herbal preparations [8]. Moreover, since numerous herbal formulations contain psoralens, their use can result in photosensitivity [28].

Zhang [29] reported a similar effect of Angelica dahuricae capsules and 8-methoxypsoralen tablets during UV-A therapy. However, when the Chinese herb preparation was being used, milder, although still of the same nature (nausea, dizziness), adverse reactions were observed in comparison with those induced by 8 -methoxypsoralen.

One should bear in mind that long-term Angelica dahuricae application may also bring about serious side effects. The study by $\mathrm{Li}$ et al. [30] revealed that patients who had been treated with Angelica dahuricae and undergone a long wave ultraviolet exposition presented some changes in the lens. The changes in the lens were also reported with long-term use of Radix angelice pubescentis-UVA therapy.

Moreover, Fu Fang Qing Dai Wan which is composed of Indigo naturalis, Rhizoma smilcis glabre,
Radix angelicae dahuricae, and Radix salviae milthiorrhiza herbs may be responsible for acute hepatitis, as documented by Verucchi et al. [4] who observed malaise, asthenia, loss of appetite, dyspepsia, nausea, vomiting, yellow sclerae, dark urine, skin jaundice and fever in their patient, approximately one month after initiating Fu Fang Qing Dai Wan therapy. Their patient also presented with significantly increased levels of hepatic enzymes as well as eosinophilia.

$\mathrm{Xu}$ et al. [31] reported a patient who developed lichen planus pemphigoides a few weeks after taking an oral preparation of Chinese herbs. The patient did not experience any relapse after she stopped taking the herbs.

Interestingly, Lim and Thirumoorthy [27] described patients who presented with severe cutaneous adverse drug reactions to traditional Chinese medicines. One of the patients had developed life-threatening toxic epidermal necrolysis after taking a 'white-coloured' TCM powder, while another was diagnosed with acute generalized exanthematous pustulosis and contact dermatitis due to the treatment of a sprained ankle. During the treatment, the patient received a TCM liniment and oral TCM medication. The liniment turned out to contain coumarin, piroxicam and salicylates, known allergens. The same authors have also described a psoriatic patient who presented with aggravated pre-existing psoriatic plaques and a new pruritic rash after taking three types of TCM tablets. Blood tests revealed atypical monocytosis and hepatitis. Later analysis of the tablets detected the presence of phenylbutazone and dexamethasone.

Other allergic reactions observed after taking TCM medicines include anaphylactic shock, allergic asthma and allergic purpura [2].

Keane et al. [10] analyzed the components of Chinese herbal creams used in the treatment of skin conditions and found that most of them, both labeled and unlabeled, contained dexamethasone in various concentrations. The authors reported a patient who experienced an exacerbation of eczema herpeticum following the use of TCM preparations. Contrary to common belief, TCM medications very often contain dangerous substances, including steroids.

\section{Conclusion}

In the light of the presented characteristics of the selected herbal remedies, and bearing in mind their ability to exert a positive effect on various pathological mechanisms involved in psoriasis, their considerable and still growing use seems reasonable. Yet, their harmful effects tend to be overlooked or underesti- 
mated. Dermatologists should be vigilant to potential hazards which may result from their patients' uncontrolled previous or current use of Chinese herbal medications.

\section{References}

1. Tse TW. Use of common Chinese herbs in the treatment of psoriasis. Clin Exp Dermatol. 2003;28:469-475.

2. Cheng CW, Bian ZX, Li YP et al. Transparently reporting adverse effects of traditional Chinese medicine interventions in randomized controlled trials. Zhong Xi Yi Jie He Xue Bao. 2008;6:881-886.

3. Koo J, Arain S. Traditional Chinese medicine for the treatment of dermatologic disorders. Arch Dermatol. 1998;134: 1388-1393.

4. Verucchi G, Calza L, Attard L, Chiodo F. Acute hepatitis induced by traditional Chinese herbs used in the treatment of psoriasis. J Gastroenterol Hepatol. 2002;17:1342-1343.

5. Chen HH, Sun CC, Tseng MP, Hsu CJ. A patch test study of 27 crude drugs commonly used in Chinese topical medicaments. Contact Dermatitis. 2003;49:8-14.

6. Cheung WI, Tse ML, Ngan $\mathrm{T}$ et al. Liver injury associated with the use of Fructus Psoraleae (Bol-gol-zhee or Bu-guzhi) and its related proprietary medicine. Clin Toxicol. 2009; 47:683-685.

7. Fleischer AB Jr, Feldman SR, Rapp SR, Reboussin DM, Exum ML, Clark AR. Alternative therapies commonly used within a population of patients with psoriasis. Cutis. 1996; 58:216-220.

8. Koo J, Desai R. Traditional Chinese medicine in dermatology. Dermatol Ther. 2003;16:98-105.

9. Lin YK, Leu YL, Yang SH, Chen HW, Wang CT, Pang JH Anti-psoriatic effects of indigo naturalis on the proliferation and differentiation of keratinocytes with indirubin as the active component. J Dermatol Sci. 2009;54:168-174.

10. Keane FM, Munn SE, Vivier AW, Higgins EM, Taylor NF. Analysis of Chinese herbal creams prescribed for dermatological conditions. West J Med. 1999;170:257-259.

11. Lin YK, Wong WR, Chang YC et al. The efficacy and safety of topically applied indigo naturalis ointment in patients with plaque-type psoriasis. Dermatology. 2007;214:155-161.

12. Lin YK, Leu YL, Huang TH et al. Anti-inflammatory effects of the extract of indigo naturalis in human neutrophils. JEthnopharmacol. 2009;125:51-58.

13. Lin YK, Chang CJ, Chang YC, Wong WR, Chang SC, Pang $\mathrm{JH}$. Clinical assessment of patients with recalcitrant psoriasis in a randomized, observer-blind, vehicle-controlled trial using indigo naturalis. Arch Dermatol. 2008;144:1457-1464.

14. Lin YK, Wong WR, Su Pang JH. Successful treatment of recalcitrant psoriasis with Indigo naturalis ointment. Clin Exp Dermatol. 2007;32:99-100.

15. Jiang J, Wu F, Lu J, Lu Z, Xu Q. Anti-inflammatory activity of the aqueous extract from Rhizoma smilacis glabrae. Pharmacol Res. 1997;36:309-314.

16. Chen L, Yin Y, Yi H, Xu Q, Chen T. Simultaneous quantification of five major bioactive flavonoids in Rhizoma smilacis glabrae by high-performance liquid chromatography. J Pharm Biomed Anal. 2007;43:1715-1720.

17. Zhang QF, Zhang ZR, Cheung HY. Antioxidant activity of Rhizoma Smilacis Glabrae extracts and its key constituent, astilbin. Food Chemistry. 2009;115:297-303.

18. Xu Q, Yuan K, Lu J, Wang R, Wu F. A new strategy for regulating immunological liver injury - effectiveness of DTH-inhibiting agents on DTH-induced liver injury to picryl chloride. Pharmacol Res. 1997;36:401-409.

19. Cai Y, Chen T, Xu Q. Astilbin suppresses collagen-induced arthritis via the dysfunction of lymphocytes. Inflamm Res. 2003;52:334-340.

20. Kang $\mathrm{OH}, \mathrm{Lee} \mathrm{GH}$, Choi $\mathrm{HJ}$ et al. Ethyl acetate extract from Angelica Dahuricae Radix inhibits lipopolysaccharide-induced production of nitric oxide, prostaglandin E2 and tumor necrosis factor-alpha via mitogen-activated protein kinases and nuclear factor-kappaB in macrophages. Pharmacol Res. 2007;55:263-270.

21. Moon TC, Jin M, Son JK, Chang HW. The effects of isoimperatorin isolated from Angelicae dahuricae on cyclooxygenase-2 and 5-lipoxygenase in mouse bone marrow-derived mast cells. Arch Pharm Res. 2008;31:210-215.

22. Hu P, Luo GA, Zhao Z, Jiang ZH. Quality assessment of radix salviae miltiorrhizae. Chem Pharm Bull. 2005;53:481-486.

23. Ling Z, Xiping Z, Fengmei Q, Ping Y, Qihui C. Protective effects of Salvia miltiorrhizae on multiple organs of rats with obstructive jaundice. Mediators Inflamm. 2009; doi: 10.1155/ /2009/602935.

24. Kim H, An C, Jung K et al. Rehmannia glutinosa inhibits tumour necrosis factor $\alpha$ and interleukin-1 secretion from mouse astrocytes. Pharmacol Res. 1999;40:171-176.

25. Tse WP, Cheng CH, Che CT, Zhao M, Lin ZX. Induction of apoptosis underlies the Radix Rubiae-mediated anti-proliferative action on human epidermal keratinocytes: implications for psoriasis treatment. Int J Mol Med. 2007;20:663-672.

26. Tse WP, Che CT, Liu K, Lin ZX. Evaluation of the anti-proliferative properties of selected psoriasis-treating Chinese medicines on cultured HaCaT cells. J Ethnopharmacol. 2006; 108:133-141.

27. Lim YL, Thirumoorthy T. Serious cutaneous adverse reactions to traditional Chinese medicines. Singapore Med J. 2005;46:714-717.

28. Ernst E. Adverse effects of herbal drugs in dermatology. $\mathrm{Br}$ J Dermatol. 2000;143:923-929.

29. Zhang GW. Treatment of psoriasis by photochemotherapy: a comparison between the photosensitizing capsule of Angelica dahurica and 8-MOP. Zhonghua Yi Xue Za Zhi. 1983;63: 16-19.

30. Li FQ, Fang FY, Li SH. A long-term follow-up of 58 cases of psoriasis treated with traditional Chinese medicine Angelica dahuricae and long wave ultraviolet. Chin J Physic Ther. 1984; 7:154-155.

31. Xu HH, Xiao T, He CD et al. Lichen planus pemphigoides associated with Chinese herbs. Clin Exp Dermatol. 2009; 34:329-332.

Submitted: 9 November, 2010 Accepted after reviews: 15 February, 2011 